M. Hashemi

\title{
The first in situ synthesis of 1,3-dioxan-5-one derivatives and their direct use in Claisen-Schmidt reactions
}

\author{
(Synthesis of dioxanones and their Claisen-Schmidt reactions)
}

https://doi.org/10.1515/hc-2019-0013

Received October 28, 2018; accepted February 26, 2019.

\begin{abstract}
A method is developed for in situ generation of 1,3-dioxan-5-one derivatives $\mathbf{2}$. These compounds are simple precursors for accessing carbohydrate structures and previously had to be produced via stepwise procedures using excessive amounts of reagents. In the present work, three different derivatives of $\mathbf{2}$ were synthesized via the reaction of trialkoxyalkanes with dihydroxyacetone dimer $\mathbf{1}$ in the presence of acetic acid as the catalyst. In the same pot, derivatives of $\mathbf{2}$ were reacted with aromatic aldehydes and $30 \mathrm{~mol} \%$ of pyrrolidine to obtain high yields of the respective bischalcones 3 within short time periods.
\end{abstract}

Keywords: 1,3-dioxan-5-one; trioses, Claisen-Schmidt reaction; dihydroxyacetone; one-pot reactions.

\section{Introduction}

Trioses are among the smallest monosaccharide biomolecules playing important roles in cellular respiration [1]. In the course of glycolysis, fructose-1,6-bisphosphate is cleaved to glyceraldehyde-3-phosphate and dihydroxyacetone phosphate (DHAP) [2]. The latter in

* Corresponding author: Mohammad M. Mojtahedi, Organic Chemistry Department, Chemistry and Chemical Engineering Research Center of Iran, P.O. Box 14335-186, Tehran, Iran, e-mail:mojtahedi@ccerci.ac.ir

M. Javad Poursharifi, Department of Chemistry, Science and Research Branch, Islamic Azad University, Tehran 1477893855, Iran M. Saeed Abaee, Organic Chemistry Department, Chemistry and Chemical Engineering Research Center of Iran, P.O. Box 14335-186, Tehran, Iran

Mohammad M. Hashemi, Department of Organic Chemistry, Faculty of Chemistry, Sharif University of Technology Tehran, 113658639, Iran turn could convert to lactic acid and pyruvic acid [3]. In the nature, DHAP can lead to more complex carbohydrates stereoselectively via enzyme-catalyzed aldol reactions [4]. The chemical equivalent to DHAP is dihydroxyacetone (1, DHA, Scheme 1) which is the only ketotriose and does not exist in enantiomeric forms and is therefore achiral [5]. DHA and its derivatives are successfully employed as $\mathrm{C}_{3}$ building blocks through synthetic manipulations for asymmetric synthesis of various compounds of interest [6].

The limitation in the chemistry of DHA is that the compound usually exists in relatively inactive dimeric form $[7,8]$ and researchers have to use its protected heterocyclic synthon, 1,3-dioxan-5-one 2 derivatives instead $[9,10]$. In this context, Enders introduced a simple and biomimetic approach for direct proline-catalyzed asymmetric synthesis of several carbohydrate structures and their related compounds through one-step aldol reactions of 2 [11-14]. Majewski reported stereodivergent synthesis of both enantiomers of glycero-allo-heptose from similar starting ketone 2 [15] and organocatalyzed synthesis of L-deoxymannojirimycin and L-deoxyidonojirimycin via syn-aldol reaction of $\mathbf{2}$ with (S)-isoserinal hydrate [16]. Interestingly, several applications of this chemistry are reported for the synthesis of natural compounds of interest such as total synthesis of ( \pm )-isophellibiline [17], ( \pm )-cortistatin J [18], and ( \pm )-erythroidines [19].

The difficulties associated with the preparation of derivatives of $\mathbf{2}$ have persuaded synthetic chemists to design and attempt new methods to obtain 2 via more convenient reactions and by performing less synthetic steps [20-23]. In the framework of our studies to develop new synthetic procedures in heterocyclic chemistry [24-27], herein we introduce a new method for in situ preparation of three various derivatives of $\mathbf{2}$ starting from $\mathbf{1}$ and trialkoxyalkanes ( $\mathrm{RCR}_{3}$ ) and acetic acid as catalyst. Then, ketones 2 are reacted in the same pot with aldehydes 


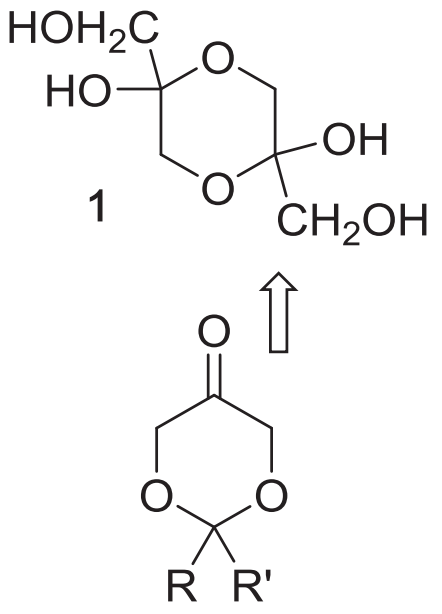

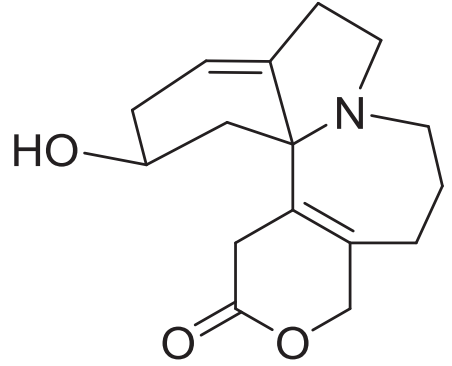

isophellibiline<smiles>CO[C@H]1C=CC2=CCN3CCC4=C(CC(=O)OC4)C23C1</smiles>

$\beta$-erythroidine

2a: $R=M e, R^{\prime}=O M e$

$2 b: R=M e, R^{\prime}=O E t$

2c: $R=H, R^{\prime}=O M e$<smiles>CN(C)[C@H]1C=CC2=CC3=CCC4(C)[C@@H](c5ccc6ccncc6c5)CC[C@H]4[C@@]34CC[C@]2(C4)O1</smiles>

Scheme 1 Important structures derived from ketones $\mathbf{2}$ (a synthon for $\mathbf{1}$ ).

to get the respective bischalcone derivatives $\mathbf{3}$ via ClaisenSchmidt condensation reactions at room temperature. The importance of chalcone functionalities in heterocyclic chemistry from synthetic $[28,29]$ and biological points of view is well documented [30,31].

\section{Results and discussion}

We first optimized the conditions for the synthesis of 2a by reacting 1 with $\mathrm{MeC}(\mathrm{OMe})_{3}$ and various catalysts (Table 1). Under the conditions reported by Müller et al [32], camphor-10-sulfonic acid (CSA) in dioxane caused $81 \%$ formation of $\mathbf{2 a}$ after $24 \mathrm{~h}$ (entry 1). Use of Lewis acids almost led to minor quantities of the desired product even at a higher temperature or a longer reaction time (entries 2-5). Acetic acid improved the result to give $80 \%$ of $2 \mathbf{a}$ at $60{ }^{\circ} \mathrm{C}$ and after a much shorter time period (entry 6). Also, less amounts of the solvent (entry 7) or the reagent (entry 8) led to comparable results. Other carboxylic acids did not behave better than $\mathrm{CH}_{3} \mathrm{COOH}$ (entries 9-10). These optimum conditions were applied successfully to prepare two other derivatives of $\mathbf{2}$ in high yields (entries 11-12).

With these results, we were persuaded to use the optimum conditions to prepare derivatives of $\mathbf{2}$ and subject them to react with an aromatic aldehyde to evaluate the possibility of the synthesis of bischalcone derivatives 3 in
Table 1 Optimization of the synthesis of $\mathbf{2}$.
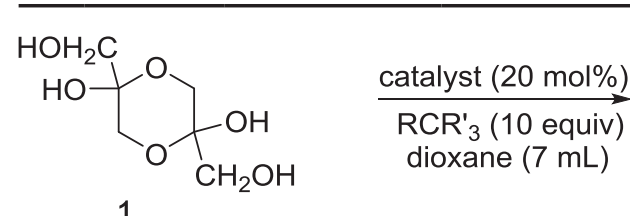<smiles>[R]C1(C)OCC(=O)CO1</smiles>

2

\begin{tabular}{|c|c|c|c|c|c|}
\hline Entry & Catalyst & Temperature $\left({ }^{\circ} \mathrm{C}\right)$ & Time (h) & Product & Yield (\%) \\
\hline 1 & CSA & 60 & 24 & $\begin{array}{l}2 a(R=M e \\
\left.R^{\prime}=O M e\right)\end{array}$ & 81 \\
\hline 2 & $\mathrm{MgBr}_{2}$ & 60 & 48 & $2 a$ & $<5$ \\
\hline 3 & $\mathrm{MgBr}_{2}$ & 80 & 72 & $2 a$ & $<5$ \\
\hline 4 & $\mathrm{LiBr}$ & 60 & 48 & $2 a$ & $<5$ \\
\hline 5 & $\mathrm{LiBr}$ & 80 & 72 & $2 a$ & $<5$ \\
\hline 6 & $\mathrm{CH}_{3} \mathrm{CO}_{2} \mathrm{H}$ & 60 & 8 & $2 a$ & 80 \\
\hline 7 & $\mathrm{CH}_{3} \mathrm{CO}_{2} \mathrm{H}^{\mathrm{b}}$ & 60 & 8 & $2 a$ & 83 \\
\hline 8 & $\mathrm{CH}_{3} \mathrm{CO}_{2} \mathrm{H}^{\mathrm{b}, \mathrm{c}}$ & 60 & 8 & $2 a$ & 83 \\
\hline 9 & $\mathrm{H}_{2} \mathrm{C}_{2} \mathrm{O}_{4}^{\mathrm{b}, \mathrm{c}}$ & 80 & 72 & $2 a$ & $<5$ \\
\hline 10 & $\mathrm{PhCO}_{2} \mathrm{H}^{\mathrm{b}, \mathrm{c}}$ & 80 & 72 & $2 a$ & $<5$ \\
\hline 11 & $\mathrm{CH}_{3} \mathrm{CO}_{2} \mathrm{H}^{\mathrm{b}, \mathrm{c}}$ & 60 & 8 & $\begin{array}{l}2 b(R=M e, \\
\left.R^{\prime}=O E t\right)\end{array}$ & 85 \\
\hline 12 & $\mathrm{CH}_{3} \mathrm{CO}_{2} \mathrm{H}^{\mathrm{b}, \mathrm{c}}$ & 60 & 8 & $\begin{array}{l}2 c(R=H \\
\left.R^{\prime}=O M e\right)\end{array}$ & $80^{\mathrm{d}}$ \\
\hline
\end{tabular}

alsolated yields. ${ }^{b}$ Dioxane $(2 \mathrm{~mL}) .{ }^{\mathrm{c}} \mathrm{MeC}(\mathrm{OMe})_{3}\left(2.0\right.$ equiv). ${ }^{\mathrm{d}} \mathrm{GC}$ yield.

the same reaction pot (Table 2). For this purpose, when monitoring of the reaction showed maximum formation of 2a, the mixture was treated with an alkaline hydroxides followed by addition of $4-\mathrm{ClC}_{6} \mathrm{H}_{4} \mathrm{CHO}$. As a result, use 
of $\mathrm{NaOH}$ (entries 1-2) or $\mathrm{KOH}$ (entries 3-4) in aqueous or solvent-free conditions gave no minor amounts of $\mathbf{3 a}$. However, when organocatalysts were used, pyrrolidine (entry 5) gave 3a in 83\% yield after $15 \mathrm{~min}$, while $\mathrm{Et}_{2} \mathrm{NH}$ (entry 6) or $\mathrm{Et}_{3} \mathrm{~N}$ (entry 7) produced $63 \%$ or $41 \%$ of the same product after similar time period.

To show the generality of the process, we synthesized various derivatives of $\mathbf{3}$ by subjecting $\mathbf{2 a}$ to react with different aldehydes bearing electron withdrawing groups (Scheme 2). Thus 3a-f were obtained in high yields. Also, the reaction with benzaldehyde itself led to the same observations and $\mathbf{3 g}$ was obtained in $80 \%$. Similarly, use of $\mathbf{2 b}$ or $\mathbf{2 c}$ produced the target products (3h-3n) in $85-95 \%$ yields. The condensation step for all reactions occurred within 15-20 min and products precipitated in the mixtures spontaneously.

Table 2 Optimization of the synthesis of $3 a$.

\begin{tabular}{lllcc}
\hline Entry & Conditions (30 mol\%) & Solvent & Time (min) & Yield (\%) $^{\mathbf{a}}$ \\
\hline 1 & $\mathrm{NaOH}$ & none & 24 & $<5$ \\
2 & $\mathrm{NaOH}$ & $\mathrm{H}_{2} \mathrm{O}$ & 24 & $<5$ \\
3 & $\mathrm{KOH}$ & none & 24 & $<5$ \\
4 & $\mathrm{KOH}$ & $\mathrm{H}_{2} \mathrm{O}$ & 24 & $<5$ \\
5 & $\mathrm{pyrrolidine}$ & none & 15 & 83 \\
6 & $\mathrm{Et}_{2} \mathrm{NH}$ & none & 15 & 63 \\
7 & $\mathrm{Et}_{3} \mathrm{~N}$ & none & 15 & 41 \\
\hline
\end{tabular}

asolated yields.

\section{Conclusion}

In summary, we succeeded to prepare derivatives of $\mathbf{2}$, which could be either isolated from the reaction mixtures or further subjected to Claisen-Schmidt reactions in the same pot. Thus various derivatives of $\mathbf{2}$ and $\mathbf{3}$ could be prepared efficiently. After the prevailing reaction conditions, the products were solidified in the reaction vessels and required no expensive and time consuming chromatographic separations. In addition, synthesis of $\mathbf{2}$ succeeded by using much less amounts of solvent and the required orthoesters.

\section{Experimental}

Melting points are uncorrected. FT-IR spectra were recorded using $\mathrm{KBr}$ disks on a Bruker Vector-22 spectrometer. NMR spectra were obtained on a FT-NMR Bruker Ultra Shield ${ }^{\mathrm{TM}}$ (500 MHz for ${ }^{1} \mathrm{H}$ and $125 \mathrm{MHz}$ for ${ }^{13} \mathrm{C}$ ) as DMSO- $\mathrm{d}_{6}$ solutions using TMS as internal standard reference. Elemental analyses were performed using a Thermo Finnigan Flash EA 1112 instrument. MS spectra were obtained on a Finnigan Mat 8430 instrument at ionization potential of $70 \mathrm{eV}$. TLC experiments were carried out on pre-coated silica gel plates using petroleum ether/EtOAc as the eluent. Chemicals and starting materials were purchased from commercial sources. Aldehydes were redistilled or recrystallized before being used. Products $\mathbf{3 a}, \mathbf{3 f}, \mathbf{3 g}$, and

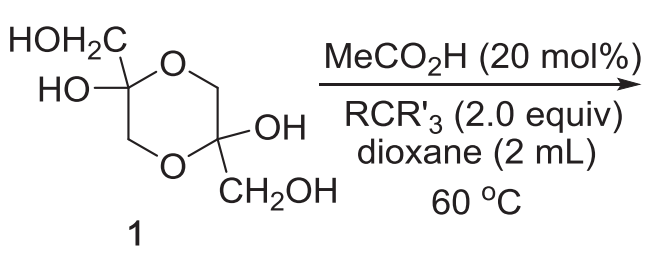

$2 a-c$

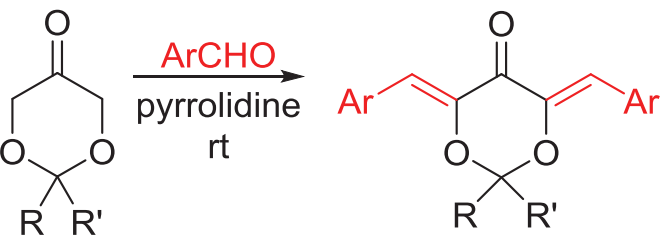

$3 \mathrm{a}(83 \%): \mathrm{R}=\mathrm{Me}, \mathrm{R}^{\prime}=\mathrm{OMe}, \mathrm{Ar}=4-\mathrm{ClC}_{6} \mathrm{H}_{4}$ $3 \mathrm{~b}(90 \%): \mathrm{R}=\mathrm{Me}, \mathrm{R}^{\prime}=\mathrm{OMe}, \mathrm{Ar}=4-\mathrm{BrC}_{6} \mathrm{H}_{4}$ $3 c(71 \%): \mathrm{R}=\mathrm{Me}, \mathrm{R}^{\prime}=\mathrm{OMe}, \mathrm{Ar}=3-\mathrm{O}_{2} \mathrm{NC}_{6} \mathrm{H}_{4}$ $3 d(70 \%): R=M e, R^{\prime}=\mathrm{OMe}, \mathrm{Ar}=4-\mathrm{NCC}_{6} \mathrm{H}_{4}$ $3 e(87 \%): R=M e, R^{\prime}=\mathrm{OMe}, \mathrm{Ar}=2,4-\mathrm{Cl}_{2} \mathrm{C}_{6} \mathrm{H}_{3}$ 3f $(81 \%): \mathrm{R}=\mathrm{Me}, \mathrm{R}^{\prime}=\mathrm{OMe}, \mathrm{Ar}=4-\mathrm{Me}_{2} \mathrm{NC}_{6} \mathrm{H}_{4}$ $3 g(80 \%): R=M e, R^{\prime}=\mathrm{OMe}, \mathrm{Ar}=\mathrm{C}_{6} \mathrm{H}_{5}$ $3 \mathrm{~h}(95 \%): \mathrm{R}=\mathrm{Me}, \mathrm{R}^{\prime}=\mathrm{OEt}, \mathrm{Ar}=4-\mathrm{ClC}_{6} \mathrm{H}_{4}$ 3i $(86 \%): \mathrm{R}=\mathrm{Me}, \mathrm{R}^{\prime}=\mathrm{OEt}, \mathrm{Ar}=4-\mathrm{BrC}_{6} \mathrm{H}_{4}$ 3j $(87 \%): \mathrm{R}=\mathrm{Me}, \mathrm{R}^{\prime}=\mathrm{OEt}, \mathrm{Ar}=2,4-\mathrm{Cl}_{2} \mathrm{C}_{6} \mathrm{H}_{3}$ $3 \mathrm{k}(81 \%): \mathrm{R}=\mathrm{Me}, \mathrm{R}^{\prime}=\mathrm{OEt}, \mathrm{Ar}=4-\mathrm{MeOC}_{6} \mathrm{H}_{4}$ 3l $(90 \%): \mathrm{R}=\mathrm{Me}, \mathrm{R}^{\prime}=\mathrm{OEt}, \mathrm{Ar}=4-\mathrm{Me}_{2} \mathrm{NC}_{6} \mathrm{H}_{4}$ $3 \mathrm{~m}(85 \%): \mathrm{R}=\mathrm{H}, \mathrm{R}^{\prime}=\mathrm{OMe}, \mathrm{Ar}=4-\mathrm{ClC}_{6} \mathrm{H}_{4}$ 3n (87\%): $\mathrm{R}=\mathrm{H}, \mathrm{R}^{\prime}=\mathrm{OMe}, \mathrm{Ar}=2,4-\mathrm{Cl}_{2} \mathrm{C}_{6} \mathrm{H}_{3}$

Scheme 2 One-pot synthesis of various derivatives of 3. 
3k were known [33, 34]. All other products were new and were characterized by analyzing their ${ }^{1} \mathrm{H}$ NMR, ${ }^{13} \mathrm{C}$ NMR, IR, and mass spectra.

\section{General procedure for the synthesis of 2}

Acetic acid (125 $\mu \mathrm{l}, 20 \mathrm{~mol} \%)$ was added dropwise to a mixture of dihydroxyacetone dimer $1(1.01 \mathrm{~g}, 5.6 \mathrm{mmol})$ in dioxane $(2 \mathrm{~mL})$, while being heated at $60{ }^{\circ} \mathrm{C}$ under argon atmosphere. After $10 \mathrm{~min}$, a trialkyl orthoacetate (23 mmol) was added to the mixture and was stirred for another $8 \mathrm{~h}$. The mixture was concentrated under reduced pressure and the residue was distilled to obtain derivatives of $\mathbf{2}$. Products $\mathbf{2 a - b}$ are known [32, 34].The structure of $\mathbf{2 c}$ is inferred from the final products ( $\mathbf{3 m}$ and $\mathbf{3 n}$ ) containing this central ring.

\section{General procedure for one-pot synthesis of 3}

Acetic acid (125 $\mu \mathrm{l}, 20 \mathrm{~mol} \%)$ was added dropwise to a mixture of dihydroxyacetone dimer $\mathbf{1}(1.01 \mathrm{~g}, 5.6 \mathrm{mmol})$ in dioxane $(2 \mathrm{~mL})$, while being heated at $60^{\circ} \mathrm{C}$ under argon atmosphere. After $10 \mathrm{~min}$, trialkyl orthoacetate $(23 \mathrm{mmol})$ was added to the mixture and the mixture was stirred for another $8 \mathrm{~h}$. TLC (petroleum ether/EtOAc 4:1) showed complete conversion of the starting materials to $\mathbf{2}$ after 8 $\mathrm{h}$. The heating source was removed and an aldehyde (18.6 mmol) and pyrrolidine (3.36 mmol, $277 \mu \mathrm{l}, 30 \mathrm{~mol} \%$ ) were added and mixing was continued at room temperature for 10-15 min. The completion of the reaction was monitored with TLC (petroleum ether/EtOAc:10:1). The product precipitated in the mixture. The crude solid product was purified by recrystallization from EtOH and solid products 3 were obtained.

\section{Spectral data of new products}

4,6-Bis((Z)-4-bromobenzylidene)-2-methoxy-2-methyl-1,3-dioxan-5-one (3b) Mp: 224-225 ${ }^{\circ} \mathrm{C}$; IR (KBr) $v$ 2940, 1578, $1464 \mathrm{~cm}^{-1}$; ${ }^{1} \mathrm{H}$ NMR (500 MHz, DMSO-d 6 ) $\delta 7.79$ (d, $J=8.5 \mathrm{~Hz}, 4 \mathrm{H}), 7.62(\mathrm{~d}, J=8.5 \mathrm{~Hz}, 4 \mathrm{H}), 6.87(\mathrm{~s}, 2 \mathrm{H})$, 3.30 (s, 3H), 1.98 (s, 3H); ${ }^{13} \mathrm{C}$ NMR (125 MHz, DMSO-d $) \delta$ 176.6, 144.5, 133.0, 132.9, 132.5, 123.4, 114.6, 113.7, 52.7, 21.1; MS (70 eV) m/z $480\left(\mathrm{M}^{+}\right)$, 404, 325, 196, 89; Anal. Calcd for $\mathrm{C}_{20} \mathrm{H}_{16} \mathrm{Br}_{2} \mathrm{O}_{4}: \mathrm{C}, 50.03 ; \mathrm{H}$, 3.36. Found: C, 50.16; H, 3.27.

2-Methoxy - 2 - meth yl- 4, 6 - bis ( (Z) - 3 nitrobenzylidene)-1,3-dioxan-5-one (3c). $\mathrm{Mp}:>250{ }^{\circ} \mathrm{C}$; IR (KBr) v 2920, 1701, 1615, 1528, $1345 \mathrm{~cm}^{-1}$; ${ }^{1} \mathrm{H}$ NMR (500
MHz, DMSO-d $\left.{ }_{6}\right) \delta 8.71(\mathrm{~s}, 2 \mathrm{H}), 8.26(\mathrm{~d}, J=8.0 \mathrm{~Hz}, 2 \mathrm{H}), 8.21$ (d, $J=8.0 \mathrm{~Hz}, 2 \mathrm{H}$ ), 7.74 (dd, $J=8.0,8.0 \mathrm{~Hz}, 2 \mathrm{H}), 7.07$ (s, 2H), 3.35 (s, 3H), 2.05 (s, 3H); ${ }^{13} \mathrm{C}$ NMR (125 MHz, DMSO-d $)$ $\delta$ 176.4, 148.6, 145.3, 137.2, 134.6, 130.9, 125.2, 124.2, 114.0, 113.7, 52.9, 21.0; MS (70 eV) m/z $412\left(\mathrm{M}^{+}\right), 381,337,163,129$; Anal. Calcd for $\mathrm{C}_{20} \mathrm{H}_{16} \mathrm{~N}_{2} \mathrm{O}_{8}:$ C, 58.26; $\mathrm{H}, 3.91 ; \mathrm{N}, 6.79$. Found: C, 58.33; H, 3.79; N, 6.90.

4,4'-((1Z,1'Z)-(2-Methoxy-2-methyl-5-oxo-1,3dioxane-4,6-diylidene)bis(methanylylidene))dibenzonitrile (3d). Mp: $248-249{ }^{\circ} \mathrm{C}$; IR ( $\left.\mathrm{KBr}\right)$ v 3053, 2228, 1603, $1279 \mathrm{~cm}^{-1}$; ${ }^{1} \mathrm{H}$ NMR (500 MHz, DMSO-d $\left.\mathrm{d}_{6}\right) 8.02(\mathrm{~d}$, $J=8.0 \mathrm{~Hz}, 4 \mathrm{H}), 7.90(\mathrm{~d}, J=8.0 \mathrm{~Hz}, 4 \mathrm{H}), 6.97(\mathrm{~s}, 2 \mathrm{H}), 3.34$

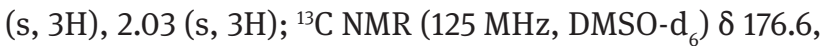
145.6, 137.6, 133.2, 131.7, 119.3, 114.1, 113.9, 111.7, 52.9, 20.9; MS (70 eV) m/z $372\left(\mathrm{M}^{+}\right), 341,297,173$, 143; Anal. Calcd for $\mathrm{C}_{22} \mathrm{H}_{16} \mathrm{~N}_{2} \mathrm{O}_{4}:$ C, 70.96; H, 4.33; N, 7.52. Found: C, 70.77; H, 4.60; N, 7.59.

4,6-Bis((Z)-2,4-dichlorobenzylidene)-2-methoxy-2methyl-1,3-dioxan-5-one (3e). Mp: 195-196 ${ }^{\circ} \mathrm{C}$; IR (KBr) v 1739, 1577, 1466, 1281, $826 \mathrm{~cm}^{-1}$; ${ }^{1} \mathrm{H}$ NMR (500 MHz, DMSOd 6$) \delta 8.12(\mathrm{~d}, J=8.0 \mathrm{~Hz}, 2 \mathrm{H}), 7.73(\mathrm{~s}, 2 \mathrm{H}), 7.51(\mathrm{~d}, J=8.0$ $\mathrm{Hz}, 2 \mathrm{H}), 7.08$ (s, 2H), 3.32 (s, 3H), 1.96 (s, 3H); ${ }^{13} \mathrm{C}$ NMR (125 MHz, DMSO-d $\mathrm{d}_{6} \delta 176.2,145.0,135.3,134.9,132.3,129.9$, 129.3, 128.3, 113.9, 109.2, 52.8, 20.7; MS (70 eV) m/z 460 $\left(\mathrm{M}^{+}\right)$, 427, 349, 186, 123; Anal. Calcd for $\mathrm{C}_{20} \mathrm{H}_{14} \mathrm{Cl}_{4} \mathrm{O}_{2}$ : C, 52.21; H, 3.07. Found: C, 52.16; H, 3.28.

4,6-Bis((Z)-4-chlorobenzylidene)-2-ethoxy-2methyl-1,3-dioxan-5-one (3h). Mp: $176-177^{\circ} \mathrm{C}$; IR (KBr) v 2965, 1595, 1485, $802 \mathrm{~cm}^{-1} ;{ }^{1} \mathrm{H}$ NMR (500 MHz, DMSO-d $\left.\mathrm{d}_{6}\right) \delta$ $7.85(\mathrm{~d}, J=8.5 \mathrm{~Hz}, 4 \mathrm{H}), 7.50(\mathrm{~d}, J=8.5 \mathrm{~Hz}, 4 \mathrm{H}), 6.87(\mathrm{~s}, 2 \mathrm{H})$, $3.61(\mathrm{q}, J=7.0 \mathrm{~Hz}, 2 \mathrm{H}), 2.0(\mathrm{~s}, 3 \mathrm{H}), 1.03(\mathrm{t}, J=7.0 \mathrm{~Hz}, 3 \mathrm{H}) ;{ }^{13} \mathrm{C}$ NMR (125 MHz, DMSO-d $) \delta 176.6,144.4,134.3,132.6,132.0$, 129.3, 114.0, 113.2, 60.9, 21.7, 15.1; MS (70 eV) m/z $404\left(\mathrm{M}^{+}\right)$, 334, 281, 181, 152; Anal. Calcd for $\mathrm{C}_{21} \mathrm{H}_{18} \mathrm{Cl}_{2} \mathrm{O}_{4}: \mathrm{C}, 62.24 ; \mathrm{H}$, 4.48. Found: C, 62.03; H, 4.59.

4,6-Bis((Z)-4-bromobenzylidene)-2-ethoxy-2-methyl-1,3-dioxan-5-one (3i). Mp: 234-235 ${ }^{\circ} \mathrm{C}$; IR (KBr) v 2923, 1597, 1481, 1283, $812 \mathrm{~cm}^{-1}$; ${ }^{1} \mathrm{H}$ NMR $\left(500 \mathrm{MHz}\right.$, DMSO-d $\left.{ }_{6}\right) \delta$ $7.79(\mathrm{~d}, J=8.5 \mathrm{~Hz}, 4 \mathrm{H}), 7.64(\mathrm{~d}, J=8.5 \mathrm{~Hz}, 4 \mathrm{H}), 6.85(\mathrm{~s}, 2 \mathrm{H})$, 3.60 (q, $J=7.0 \mathrm{~Hz}, 2 \mathrm{H}), 1.99$ (s, 3H), $1.02(\mathrm{t}, J=7.0 \mathrm{~Hz}, 3 \mathrm{H}) ;{ }^{13} \mathrm{C}$ NMR (125 MHz, DMSO-d ) $\delta$ 176.6, 144.5, 132.9, 132.3, 123.1, 114.1, 113.2, 112.9, 61.0, 21.6, 15.1; MS (70 eV) m/z $494\left(\mathrm{M}^{+}\right)$, 424, 269, 198, 149; Anal. Calcd for $\mathrm{C}_{21} \mathrm{H}_{18} \mathrm{Br}_{2} \mathrm{O}_{4}$ : C, 51.04; H, 3.67. Found: C, 50.92; H, 3.77.

4,6-Bis((Z)-2,4-dichlorobenzylidene)-2-ethoxy-2methyl-1,3-dioxan-5-one (3j). Mp: $205-206{ }^{\circ} \mathrm{C}$; IR (KBr) v 2923, 1577, 1464, $1126 \mathrm{~cm}^{-1}$; ${ }^{1} \mathrm{H}$ NMR (500 MHz, DMSO-d 6 ) $\delta$ $8.11(\mathrm{~d}, J=8.5 \mathrm{~Hz}, 2 \mathrm{H}), 7.70$ (s, 2H), $7.50(\mathrm{~d}, J=8.5 \mathrm{~Hz}, 2 \mathrm{H})$, $7.06(\mathrm{~s}, 2 \mathrm{H}), 3.61(\mathrm{q}, J=7.0 \mathrm{~Hz}, 2 \mathrm{H}), 1.96(\mathrm{~s}, 3 \mathrm{H}), 1.04(\mathrm{t}, J=7.0$ $\mathrm{Hz}, 3 \mathrm{H}) ;{ }^{13} \mathrm{C}$ NMR $(125 \mathrm{MHz}$, DMSO-d 6 ) $\delta 176.4,145.2,135.3$, 134.8, 132.3, 129.9, 129.4, 128.4, 113.6, 108.9, 61.3, 21.5, 15.3; 
MS (70 eV) m/z $474\left(\mathrm{M}^{+}\right), 404,217,186,123$; Anal. Calcd for $\mathrm{C}_{21} \mathrm{H}_{16} \mathrm{Cl}_{4} \mathrm{O}_{4}$ : C, 53.20; H, 3.40. Found: C, 53.36; H, 3.38.

4,6-Bis((Z)-4-(dimethylamino)benzylidene)-2ethoxy-2-methyl-1,3-dioxan-5-one (31). Mp: 230-231 ${ }^{\circ} \mathrm{C}$; IR (KBr) v 1591, 1527, $1127 \mathrm{~cm}^{-1}$; ${ }^{1} \mathrm{H}$ NMR (500 MHz, DMSOd $) \delta 7.69(\mathrm{~d}, J=9.0 \mathrm{~Hz}, 4 \mathrm{H}), 6.76(\mathrm{~d}, J=9.0 \mathrm{~Hz}, 4 \mathrm{H}), 6.75$ (s, 2H), 3.58 (q, $J=7.0 \mathrm{~Hz}, 2 \mathrm{H}), 2.98$ (s, 12H), 1.94 (s, 3H), 1.02 (t, $J=7.0 \mathrm{~Hz}, 3 \mathrm{H}) ;{ }^{13} \mathrm{C}$ NMR (125 MHz, DMSO-d $) \delta 175.7$, 151.0, 141.9, 132.6, 121.0, 116.1, 112.5, 112.3, 60.2, 40.1, 22.1, 15.3; MS (70 eV) m/z $422\left(\mathrm{M}^{+}\right), 306,266,205,161$; Anal. Calcd for $\mathrm{C}_{25} \mathrm{H}_{30} \mathrm{~N}_{2} \mathrm{O}_{4}$ : C, 71.07; $\mathrm{H}, 7.16 ; \mathrm{N}, 6.63$. Found: C, 71.15; H, 7.27; N, 6.52 .

4,6-Bis((Z)-4-chlorobenzylidene)-2-methoxy-1,3dioxan-5-one (3m). Mp: $155-156{ }^{\circ} \mathrm{C}$; $\mathrm{IR}(\mathrm{KBr})$ v 2934, 1606, 1578, 1464, $825 \mathrm{~cm}^{-1}$; ${ }^{1} \mathrm{H}$ NMR (500 MHz, DMSO-d $) \delta 7.87$ (d, $J=8.5 \mathrm{~Hz}, 4 \mathrm{H}), 7.50$ (d, $J=8.5 \mathrm{~Hz}, 4 \mathrm{H}), 6.93$ (s, 2H), 6.55 (s, 1H), 3.43 (s, 3H); ${ }^{13} \mathrm{C}$ NMR (125 MHz, DMSO-d $) \delta 176.4$, 144.1, 134.5, 132.7, 131.8, 129.3, 114.9, 107.2, 54.3; MS (70 eV) m/z $376\left(\mathrm{M}^{+}\right)$, 315, 281, 225, 152; Anal. Calcd for $\mathrm{C}_{19} \mathrm{H}_{14} \mathrm{Cl}_{2} \mathrm{O}_{4}$ : C, 60.50; H, 3.74. Found: C, 60.66; H, 3.85.

4,6-Bis((Z)-2,4-dichlorobenzylidene)-2-methoxy1,3-dioxan-5-one (3n). Mp: 186-187 ${ }^{\circ} \mathrm{C}$; IR (KBr) v 2897, 1577, 1464, 1268, $824 \mathrm{~cm}^{-1}$; ${ }^{1} \mathrm{H}$ NMR (500 MHz, DMSO-d $) \delta$ 8.12 (d, $J=8.5 \mathrm{~Hz}, 2 \mathrm{H}), 7.72$ (s, 2H), 7.52 (d, $J=8.5 \mathrm{~Hz}, 2 \mathrm{H})$, 7.11 (s, 2H), 6.58 (s, 1H), 3.43 (s, 3H); ${ }^{13} \mathrm{C}$ NMR (125 MHz, DMSO-d $)_{6} \delta 176.2,144.9,135.3,135.0,132.3,129.9,129.3$, 128.3, 109.9, 107.3, 54.5; MS (70 eV) m/z $446\left(\mathrm{M}^{+}\right)$, 409, 349, 186, 123; Anal. Calcd for $\mathrm{C}_{19} \mathrm{H}_{12} \mathrm{Cl}_{4} \mathrm{O}_{4}$ : C, 51.16; H, 2.71. Found: C, 51.27; H, 2.86.

Acknowledgment: The Research Council at CCERCI (Grant \# 96-112) is acknowledged for financial support of this work.

\section{References}

[1] Jeremy, M. B.; Tymoczko, J. L.; Stryer, L. Biochemistry; 5th ed., Freemanm: New York, 2002.

[2] Robina, I.; Vogel, P. Synthesis of monosaccharides and analogues. part 1. applications of chemoenzymatic methods and organocatalysis. Chim. Oggi 2007, 25, 65-71.

[3] Lux, S.; Siebenhofer, M. Synthesis of lactic acid from dihydroxyacetone: use of alkaline-earth metal hydroxides. Catal. Sci. Technol. 2013, 3, 1380-1385.

[4] Suri, J. T.; Ramachary, D. B.; Barbas, C. F. Mimicking dihydroxyacetone phosphate-utilizing aldolases through organocatalysis: a facile route to carbohydrates and aminosugars. Org. Lett. 2005, 7, 1383-1385.

[5] Rao, V. S. R.; Qasba, P. K.; Balaji, P. V.; Chandrasekaran, R. Conformation of carbohydrates; Harwood Academic Publishers: Amsterdam, 1998.
[6] Enders, D.; Voith, M.; Lenzen, A. The dihydroxyacetone unit-a versatile C(3) building block in organic synthesis. Angew. Chem. Int. Ed. Engl. 2005, 44, 1304-1325.

[7] Gardiner, D. The dimeric forms of some $\alpha$-hydroxycarbonyl compounds. Carbohydr. Res. 1966, 2, 234-239.

[8] Kobayashi, Y.; Igarashi, T.; Takahashi, H.; Higasi, K. Infrared and raman studies of the dimeric structures of 1,3-dihydroxyacetone, d(+)- and dl-glyceraldehyde. J. Mol. Struct. 1976, 35, 85-99.

[9] Feng, J.; Liu, B. Formal carbo [3+3] annulation and its application in organic synthesis. Tetrahedron Lett. 2015, 56, 1474-1485.

[10] Carlsen, P. H. J.; Søerbye, K.; Ulven, T. Aasbøe, K. Synthesis of benzylidene-protected dihydroxyacetone. Acta. Chem. Scand. 1996, 50, 185-187.

[11] Grondal, C.; Enders, D. Direct asymmetric organocatalytic de novo synthesis of carbohydrates. Tetrahedron 2006, 62, 329-337.

[12] Enders, D.; Grondal, C. Direct organocatalytic de novo synthesis of carbohydrates. Angew. Chem. Int. Ed. Engl. 2005, 44, 1210-1212.

[13] Enders, D.; Jegelka, U. 1,3-Dioxan-5-one as C3-building block for the diastereo- and enantioselective synthesis of $\mathrm{C}_{5}$ - to C9-deoxy sugars using the SAMP-/RAMP-hydrazone method. Tetrahedron Lett. 1993, 34, 2453-2456.

[14] Enders, D.; Narine, A. A. Lessons from nature: biomimetic organocatalytic carbon-carbon bond formations. J. Org. Chem. 2008, 73, 7857-7870.

[15] Palyam, N.; Niewczas, I.; Majewski, M. Stereodivergent synthesis of d,d-and l,l-glycero- $\beta$-allo-heptopyranoses on a dioxanone scaffold. Synlett 2012, 23, 2367-2370.

[16] Palyam, N.; Majewski, M. Organocatalytic syn-aldol reactions of dioxanones with (S)-isoserinal hydrate: synthesis of l-deoxymannojirimycin and l-deoxyidonojirimycin. J. Org. Chem. 2009, 74, 4390-4392.

[17] Funk, R. L.; Belmar, J. Total synthesis of ( \pm )-isophellibiline. Tetrahedron Lett. 2012, 53, 176-178.

[18] Nilson, M. G.; Funk, R. L. Total synthesis of ( \pm )-cortistatin J from furan. J. Am. Chem. Soc. 2011, 133, 12451-12453.

[19] He Y.; Funk, R. L. Total syntheses of ( \pm )- $\beta$-erythroidine and $( \pm)$-8-oxo- $\beta$-erythroidine by an intramolecular Diels-Alder cycloaddition of a 2-amidoacrolein. Org. Lett. 2006, 8, 3689-3692.

[20] Enders, D.; Voith, M.; Ince, S. J. Preparation and reactions of 2,2-dimethyl-1,3-dioxan-5-one-SAMP-hydrazone: a versatile chiral dihydroxyacetone equivalent. Synthesis 2002, 34, 1775-1779.

[21] Peukert, S.; Giese, B. The pivaloylglycol anchor group: a new platform for a photolabile linker in solid-phase synthesis. J. Org. Chem. 1998, 63, 9045-9051.

[22] Majewski, M.; Gleave, D. M.; Nowak, P. 1,3-Dioxan-5-ones: synthesis, deprotonation, and reactions of their lithium enolates. Can. J. Chem. 1995, 73, 1616-1626.

[23] Smithson, T. L.; Ibrahim, N.; Wieser, H. Cyclohexanones: evidence of chair inversion and estimate for barriers to planarity from the far-infrared spectra. Can. J. Chem. 1983, 61, 1924-1932.

[24] Abaee, M. S.; Mojtahedi, M. M.; Hamidi, V.; Mesbah, A. W.; Massa, W. The first synthesis of bis (arylmethylidene) dioxan-5-ones: potential scaffolds to access vicinal tricarbonyl derivatives. Synthesis 2008, 40, 2122-2126. 
[25] Mojtahedi, M. M.; Mehraban, M.; Darvishi, K.; Abaee, M. S. Ultrasound mediated synthesis of dihydropyrano $[3,2-d]$ $[1,3]$ dioxin-7-carbonitrile derivatives in $\mathrm{H}_{2} \mathrm{O} / \mathrm{EtOH}$ medium. Heterocycl. Commun. 2017, 23, 91-95.

[26] Abaee, M. S.; Akbarzadeh, E.; Shockravi, A.; Mojtahedi, M. M.; Mehraki, E.; Khavasi, H. R. Three-component anti selective Mannich reactions in a tetrahydro-4-pyranone system by using PDAG-Co catalyst. Heterocycl. Commun. 2014, 20, 123-128.

[27] Mojtahedi, M. M.; Pourabdi, L.; Abaee, M. S.; Jami, H.; Dini, M.; Halvagar, M. R. Facile one-pot synthesis of novel orthoaminocarbonitriles and dicyanoanilines fused to heterocycles via pseudo four-component reactions. Tetrahedron 2016, 72, 1699-1705.

[28] Li, Z.; Song, W.; He, J.; Du, Y.; Yang, J. Synthesis of 4-arylethyl6-arylpyrimidine-2-thiols through aza-Michael addition/ nucleophilic addition/aromatization tandem reactions. Heterocycl. Commun. 2018, 24, 23-26.

[29] El-Hussieny, M.; Yosef, H. A. A.; Mahran, M. R. H.; Ibrahim, N. M. Reactions of ferrocenyl chalcones with hydrazines and active methylene compounds. Heterocycl. Commun. 2016, 22, 69-77.

[30] Singh, P.; Anand, A.; Kumar, V. Recent developments in biological activities of chalcones: a mini review. Eur. J. Med. Chem. 2014, 85, 758-777.

[31] Arasavelli, A. M.; Ganapavarapu, V.; S. Vidavalur, S. Design, synthesis, and anticancer activity of novel aryl/ heteroaryl chalcone derivatives. Heterocycl. Commun. 2016, 22, 1-5.

[32] Müeller, S. N.; Batra, R.; Senn, M.; Giese, B.; Kisel, M.; Shadyro, 0 . Chemistry of C-2 glyceryl radicals: indications for a new mechanism of lipid damage. J. Am. Chem. Soc. 1997, 119, 2795-2803.

[33] Mojtahedi, M. M.; Darvishi, K.; Abaee, M. S.; Halvagar, M. $R$. Synthesis and fluorescence studies of novel bisarylmethylidene derivatives of 2-methoxy-2-methyl-1, 3-dioxan-5-one. Can. J. Chem. 2017, 95, 785-791.

[34] Rudolph, T.; Buehle, P.; Rosskopf, R. US Patent 2013/0309184 A1, 2013. 